\title{
Medio ambiente, pedagogía ciudadana y derechos colectivos
}

Environment, citizen pedagogy and collective rights

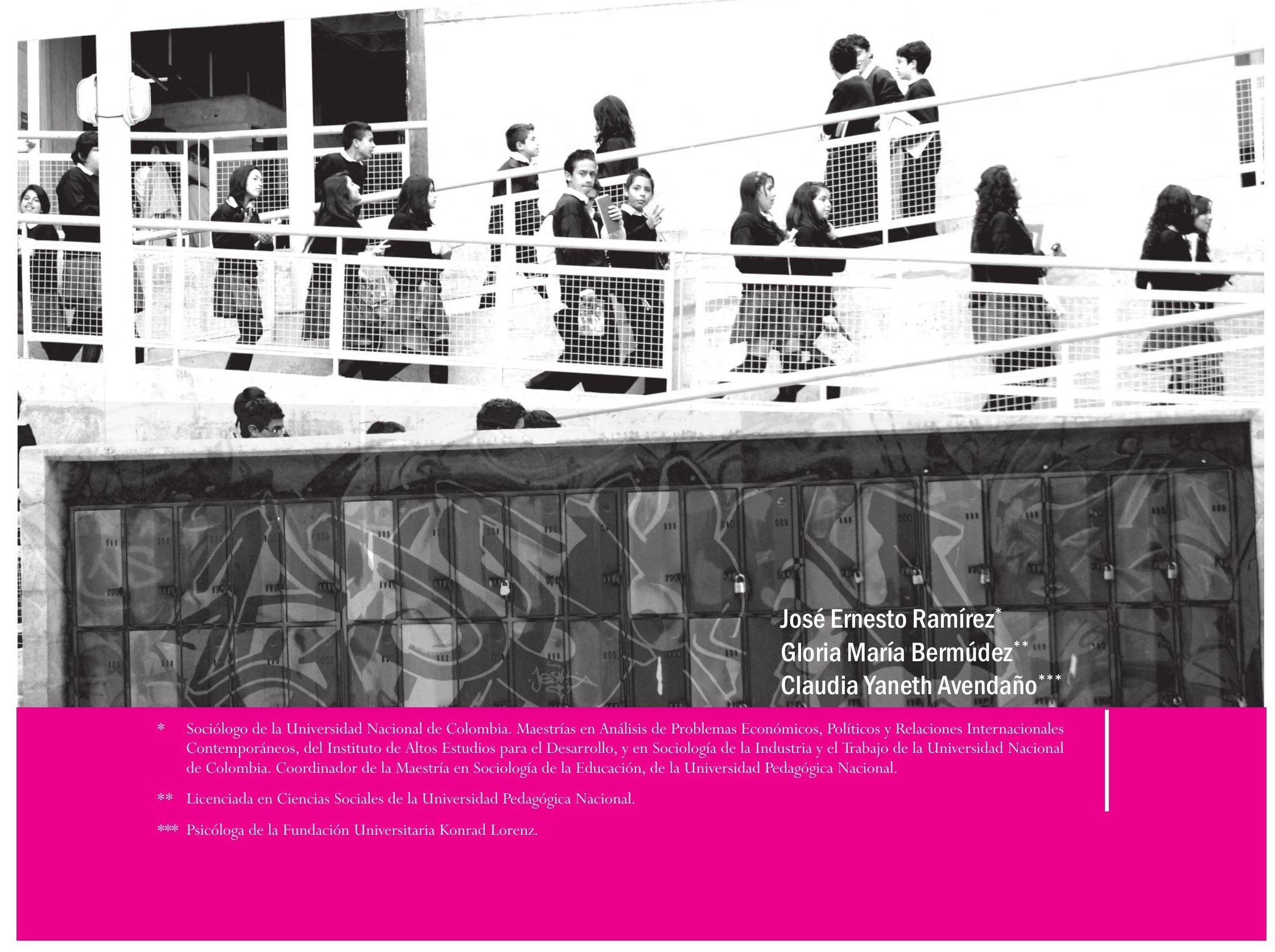


Se reflexiona en torno a la utilización del medio ambiente en los procesos pedagógicos de la escuela, para la formación de los valores de la cultura ciudadana desde los primeros años escolares.

Específicamente, a manera de aporte, se precisan las articulaciones metodológicas desde tres perspectivas:

1. Ordenar los elementos de carácter teórico y los saberes pedagógicos sobre el medio ambiente que han tomado relevancia en Colombia y en el ámbito local.

2. Vincular la conceptualización de los ciclos académicos y la caracterización de los rasgos psicopedagógicos de los escolares de diversos ciclos, en la perspectiva de identificar los conocimientos mínimos con los que se buscaría dotarlos a través de un proceso innovativo.

3. Proponer la emergencia y fortalecimiento de una nueva perspectiva de la formación en competencias ciudadanas, entendida como derechos colectivos y sus incidencias no sólo en el diseño curricular de la educación básica, sino extensible a los procesos no formales y de las otras modalidades de cultura política.

El artículo está organizado en las siguientes secciones: primera: de la teoría general al currículo aplicado del medio ambiente en el contexto de Bogotá; segunda: apropiación de nuevos saberes pedagógicos en la perspectiva de los derechos colectivos ambientales; tercera: perspectivas de innovación y transformación de la educación ambiental escolar en Bogotá. Finalmente se presentan algunas conclusiones y referencias documentales.

Palabras claves: medio ambiente, derecho colectivo, cultura ciudadana, pedagogía ciudadana.

Abstract Reflections around the use of the environment in the pedagogical process of the school, for the formation of the citizen culture values since the first scholar years. Specifically as a contribution, the methodological articulations are viewed from three perspectives:

1. To straighten the theoretical character elements and the pedagogical knowledge on the environment which have been relevant in Colombia and in its local sphere.

2. To link the planning of the academic cycles and the characterization of the psycho pedagogical characteristics of the students of diverse cycles, in view to identify the minimum knowledge that would be good to give them through an innovative process.

3. To propose the emergency and the fortification of a new perspective of the formation in citizen competitions, understood as collective rights and their incidences not only in the curricular design of the basic education but extended to the non formal processes and the other modalities of political culture.

The article is organized in the following way: First: from the general theory to the applied curriculum of the environment in the context of Bogotá. Second: Appropriation of new pedagogical knowledge in the perspective of the environmental collective rights. Third: Perspective of innovation and transformation of the scholar environmental education in Bogotá. Finally, some conclusions and documents references are given.

Key words: environment, collective right, citizen culture, citizen pedagogy. 


\section{De la teoría general al currículo aplicado del medio ambiente en el contexto de Bogotá}

En esta sección se desarrollan las siguientes aproximaciones: 1) a la crítica del modelo actual de sostenibilidad como enfoque de la problemática ambiental; 2) a la caracterización y elementos de continuidad entre la crisis planetaria, nacional y local, con alguna mención de su normatividad; 3) a la configuración actual de la educación ambiental también en los niveles desagregados. 


\section{Crítica del modelo actual de sostenibilidad como enfoque de la problemática ambiental}

Antes que nada se debe diferenciar el ambiente, interpretado en forma simple como el conjunto de relaciones y efectos de las mismas entre todos los seres vivos y los factores asociados, y la crisis ambiental o problemática ambiental. El ambiente constituye un campo de estudio profundo de la ecología, disciplina que ha aportado los mayores elementos de comprensión al problema en la medida que intenta explicar las leyes generales de los seres vivos.

Diversas perspectivas han contribuido a la acumulación de un saber universal sobre el tema, en un grado de complejidad cada día mayor. Respecto a la caracterización de la crisis, en extremo definida como ecocidio planetario (Vega, 2008), se señala que una de las regulaciones más importantes en un sistema vivo es siempre la existencia de excedentes, en virtud de la que no toda la producción del metabolismo autótrofo es consumida por el heterótrofo. Con la intervención humana en las cadenas alimenticias, y transformando tecnológicamente la materia, en una irrupción reiterada en la regulación "natural", los mecanismos naturales no logran compensar el sistema y tampoco los culturales logran actuar de manera anticipada.

La comprensión del carácter indispensable del equilibrio vital para la subsistencia humana ha dado paso a la fijación de principios de filosofía política fundados en la premisa de la resiliencia ecosistémica (Ángel, 2007), que adoptando la expresión de manejo sostenible propone no usar más de lo que le debe quedar al sistema para su normal funcionamiento. Dado que, a pesar de los grandes desarrollos científicos, subsiste gran desconocimiento sobre los principios de regulación del orden natural, la premisa y condicionante del uso sostenible es el enfoque de precaución.

El enfoque ambientalista ha terminado configurando una propuesta de tipo político que de manera práctica ha avanzado en las últimas tres décadas. Por su parte la "ética de la conservación" se ha adaptado en la visión del "desarrollo sustentable", término que se ha venido revelando insuficiente para guiar una acción económica y política consensuada, haciendo que el desarrollo sostenible, dos décadas después de su declaración en 1987, siga siendo una meta inalcanzada, mientras que la inequidad y la pobreza han aumentado y los recursos del medio ambiente, de los cuales dependen los seres vivos y aquellos de los que depende la economía actual, muestran señales de agotamiento, degradación o extinción. El modelo de desarrollo que se concibe desde el crecimiento económico explica el fracaso del desarrollo y bienestar social con base en las limitaciones tecnológicas, los recursos del medio ambiente y la capacidad del medio ambiente para absorber los efectos de la actividad humana. De esta manera, se plantea la posibilidad de mejorar la tecnología y la organización social de forma que el medio ambiente pueda recuperarse al mismo ritmo que es afectado por la actividad humana (Daly, 2003). 
La premisa anterior concibe un planeta ilimitado, no limitado, y unos recursos naturales que pueden expandirse. Sin embargo, como lo plantea Daly (2003), la Tierra, como un todo, es un estado fijo, ni su superficie ni su masa están en proceso de contracción o expansión, la entrada y salida de energía son equivalentes, y el material importado del espacio y su exportación son equivalentes pero en realidad insignificantes. Pero un estado fijo no significa un estado estático, adentro se dan toda clase de procesos dinámicos y de intercambios de materia y energía en los procesos ecológicos. Así, entre más se acerque la economía a la escala completa del globo terráqueo, más tendrá que asemejarse al comportamiento físico de la Tierra. Este comportamiento es un estado fijo, es decir, un sistema que permita el desarrollo cualitativo pero sin agregar crecimiento cuantitativo. El crecimiento es más de las mismas cosas, desarrollo es la misma cantidad de mejores cosas.

El modelo económico actual busca que los países ricos sigan creciendo tan rápido como sea posible para crear mercado y acumular más capital para invertir en países pobres. La economía de estado fijo (Daly, 2003) propone que los ricos reduzcan el crecimiento de sus rendimientos para librar recursos y espacio ecológico para ser usado por los pobres, mientras que sus esfuerzos internos los concentren en desarrollo, en avances e innovaciones técnicas y sociales que puedan compartir de manera gratuita con los países pobres. De no ser así, los ecosistemas no son capaces de proveer las fuentes ni los sumideros para los excedentes (rendimientos) metabólicos necesarios para sustentar la actual economía y, por supuesto, menos los de una economía creciente.

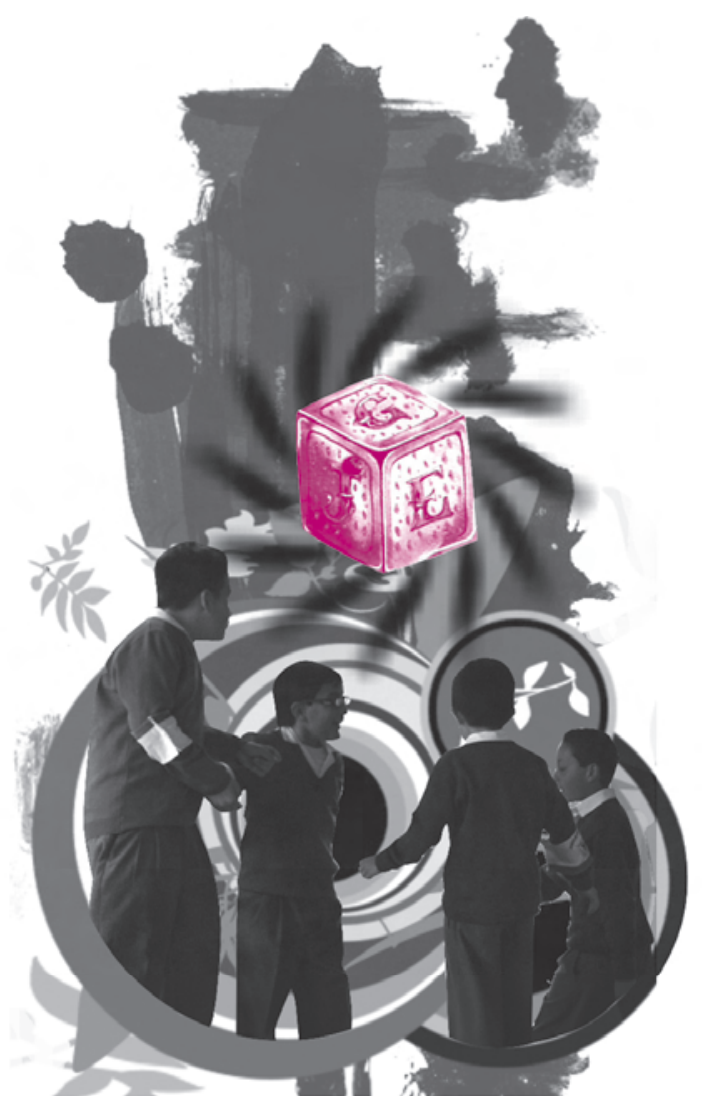




\section{Caracterización y elementos de continuidad entre la crisis planetaria, nacional y local, con alguna mención de su normatividad}

Los principales referentes de causalidad de los problemas ambientales son propuestos en las narrativas dominantes como derivados de factores demográficos — crecimiento desproporcionado respecto a la distribución espacial de los territorios y los recursos para la satisfacción de estándares de vida - y tecnológicos — consumo de energía fósil, sobreproducción de desechos cada vez más tóxicos, presión sobre los recursos hídricos-, con las conocidas consecuencias en el equilibrio de los ecosistemas: sustitución de los bosques por deforestación o por hatos, incremento de la concentración de la propiedad de la tierra, desaparición de la biodiversidad y sobrecontaminación atmosférica.

En Colombia, las grandes ciudades enfrentan problemáticas relacionadas con altos niveles de contaminación del aire y del agua; mala disposición de desechos industriales, comerciales, domésticos; y el manejo de aguas residuales. Aunque el efecto invernadero aún no reviste el dramatismo que se vive en otras regiones del planeta, sí son notorios los efectos de la emisión de sustancias dañinas en la capa de ozono, que no se detiene aún, y los efectos del cambio climático revelados en variaciones en el régimen de lluvias e incremento de la temperatura promedio. Mucho más visible es la pérdida de los casquetes de hielo en las altas montañas de los nevados, y se considera amenazante el incremento del nivel del mar por el descongelamiento polar. La disponibilidad de agua dulce se empieza a ver seriamente afectada por la contaminación de los cursos de agua, en contraste con la deficiencia de la acción de contingencia sobre la ocupación de los márgenes de los ríos, para la conservación de cuencas y para la reparación ambiental. En las extensas porciones rurales se evidencian las consecuencias ambientales del conflicto social, como pérdida del hábitat, explotación descontrolada de especies vegetales y animales, y en regiones como la Andina es ostensible el mayor nivel de degradación ambiental. Desafortunadamente las "reservas naturales" también son sometidas a procesos de "privatización", presencia de inversiones multinacionales y saqueo de la enorme riqueza de "capital natural" que distinguió el suelo colombiano.

Bogotá, como capital del país, alberga más del $15 \%$ de la población nacional, y como resultado de la alta concentración demográfica, aunada a la falta de planificación en el uso del territorio, las principales amenazas para el ambiente en el Distrito están representadas en el deterioro de las cuencas hidrográficas, la transformación de los ecosistemas originarios y la contaminación de la atmósfera, de los cauces de agua y sonora (Montoya Villarreal y Manrique, 2005). El desequilibrio socioeconómico hace que la población con mayor vulnerabilidad ambiental se concentre en las localidades de subnormalidad y periferia como Ciudad Bolívar, Usme, San Cristóbal y Bosa. La dinámica expansiva de la actividad constructora legal e ilegal irrespeta áreas sagradas para la ciudad como los humedales, los Cerros Orientales y las cuencas subterráneas. El 65\% de la contaminación atmosférica se debe al tránsito vehicular que aún no 
queda sometido a disposiciones ambientales rígidas y eficaces. La contaminación del río Bogotá es la principal problemática ambiental que enfrenta la ciudad, expresada en el deterioro de la calidad de sus aguas para abastecimientos y riegos y en la generación de patologías que pueden llegar a tener características epidémicas.

Como se deriva de una comparación en la desagregación de los problemas ambientales, la situación de Bogotá es tan cercana (sobreconcentración poblacional, emisión de gases contaminantes, daños en las fuentes hídricas, altos niveles de ruido, etc.) de la que afecta al país y al mundo, como específica en lo que concierne a sus formas de relación entre territorio, dotación de capital natural y procesos de contención emprendidos con apoyo en la institucionalización de políticas ambientales y la sensibilización cultural.

Mirando la normatividad ambiental nacional se destaca que Colombia desde los años setenta del siglo XX se adhirió a las corrientes internacionales de regulación sobre temas como diversidad biológica, cambio climático, Agenda 21, Protocolo de Montreal por la capa de ozono, Convención sobre el Comercio Internacional de Especies Amenazadas de Fauna y Flora Silvestres, entre otros (IDEAm et al., 2002). Más recientes son: la aprobación del Convenio de Viena en 1990, y de la Convención sobre el Cambio Climático en 1994ํ․ En el Código de Recursos Naturales Renovables de 1974 se estableció por primera vez el que toda persona tiene derecho a disfrutar de ambiente sano (Sánchez Pérez, 2002). Luego, en la Constitución de 1991 se determinaron disposiciones importantes para la protección y conservación del ambiente en Colombia, asignando deberes ambientales al Estado y a los ciudadanos. También se responsabilizó a las organizaciones del Estado de implementar mecanismos que vinculen a la sociedad civil con el diseño y la implementación de políticas ambientales. En 1993 se creó el Ministerio del Medio Ambiente, ente coordinador del Sistema Nacional Ambiental (SINA), el cual fue definido como el conjunto de orientaciones, normas, actividades, recursos, programas e instituciones que permiten la puesta en marcha de los principios generales ambientales orientados hacia el desarrollo sostenible (Viceministerio del Medio Ambiente, 2000). También se crearon 16 corporaciones autónomas regionales y se reestructuraron las 18 existentes (IDEAM et al., 2002). Desde entonces los Planes Nacionales de Desarrollo han acogido capítulos de "crisis ambiental", "sostenibilidad ambiental" y contemplado la educación como herramienta fundamental. Es así como en un decreto de 1994 se instituyeron los PRAE (proyectos ambientales escolares) dentro del currículo de la educación básica y media. En 1999 se crearon como parte del SINA cinco institutos de investigación especializados en el conocimiento de la realidad natural del país, que con las autoridades urbanas y la unidad de Parques Nacionales Naturales, y en asocio con otros organismos de soporte transectorial, territorial y social, conforman la actual infraestructura del campo ambiental colombiano.

En el caso de Bogotá, se ha definido una estructura ecológica principal con un componente nacional y regional (el Parque Nacional Natural de Sumapaz, el bosque protector de los Cerros Orientales y la Reserva Forestal de Sierra Morena), y otro componente urbano en el que se definen dos subsistemas: el de montaña u orográfico, y el hídrico. La estructura ecológica rural 
la conforma el sistema de áreas protegidas, que incluye los subsistemas naturales y seminaturales del Distrito. El Parque Ecológico Distrital contiene 5 montañas y 12 humedales, además de 3 santuarios distritales de flora y fauna. La Reserva Forestal Distrital está clasificada en Ecosistema Estratégico para la Preservación, con 27 reservas, y en Ecosistema Estratégico para la Restauración (EER), con 16 zonas de reserva y recuperación. En 2002 el área verde por habitante era de 4,7 m², que se espera incrementar a 9,3 $\mathrm{m}^{2}$ por habitante, luego de la expansión de 22 parques planeados.

\section{Configuración actual de la educación ambiental}

El advenimiento del siglo XXI produjo reflexiones y demandas a los sistemas educativos mundiales que se concretaron en reformas para afrontar los nuevos retos y reorientar concepciones, objetivos y prioridades en todos los niveles. Éstas incluyen significados modernos en aspectos legales, pedagógicos y didácticos, así como en las formas de ejercer la administración y gestión. La actual realidad educativa no se plantea al margen de las incertidumbres y paradojas del escenario internacional que se mueve entre la globalización, la identidad cultural y la búsqueda de una mayor equidad social, sino que revaloriza su principal función: la de forjar mejores ciudadanos, responsables y conscientes de sus derechos y deberes constitucionales. Las tendencias y retos educativos para el siglo XXI se han hecho visibles principalmente desde las reflexiones y marcos de acción propuestos en la Cumbre de Jomtien (1990), el Foro Mundial de Dakar (2000) y la Conferencia Internacional de Educación (2001), en donde se precisaron recomendaciones para un cambio en las políticas y en las prácticas educativas, a fin de progresar juntos en la construcción de una educación más pertinente para alcanzar todos los derechos y el desarrollo humano (Unesco, 2001). La Conferencia Mundial sobre Educación para Todos, en Jomtien ${ }^{2}$, hace un llamado hacia una mirada integral de la educación sobre necesidades básicas de aprendizaje y proclama que todas las personas deben beneficiarse de una educación básica que comprenda tanto las herramientas esenciales para el aprendizaje en su dimensión instrumental-técnica ${ }^{3}$ como los contenidos básicos de aprendizaje ${ }^{4}$ en su dimensión ético-política. El Foro Mundial de Dakar, ${ }^{5}$ por su parte, ratifica que la calidad de la educación es y debe ser el centro de la "educación para todos" y manifiesta un compromiso colectivo para actuar y cumplir los objetivos y finalidades para el año 2015. En este Foro se plantean estrategias que se traducen en programas para favorecer el entendimiento y la tolerancia como valores sociales y éticos, así como el desarrollo de acciones para modificar actitudes, valores y prácticas.

2 Jomtien, Tailandia (1990). La declaración adoptada por la Conferencia Mundial sobre Educación para Todos, proclama que toda persona debe beneficiarse de una educación básica para satisfacer sus necesidades de aprendizaje, y se destacan los siguientes componentes: la universalización del acceso a la educación y el fomento de la equidad; la atención prioritaria al aprendizaje; la ampliación de los medios y del alcance de la educación básica; la mejora del ambiente para el aprendizaje y el fortalecimiento de la concertación de acciones que buscan unir equidad, desarrollo y conocimiento. La equidad se considera el elemento central que reorganiza el nuevo papel de la educación y articula, como factor de equilibrio social, el progreso técnico, la transformación productiva y la educación.

3 Herramientas esenciales para el aprendizaje (lectura y escritura, expresión oral, aritmética y resolución de problemas.

4 Contenidos básicos de aprendizaje (conocimientos teóricos y prácticos, valores y actitudes).

5 Foro Mundial de Educación, celebrado en Dakar, Senegal, en abril de 2000. 


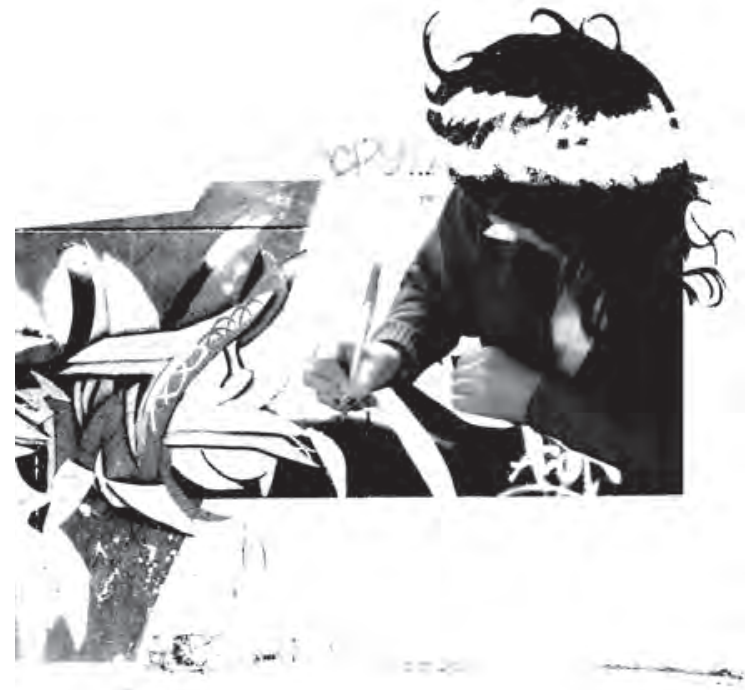

El Informe Delors ${ }^{6}$ reivindica el componente éticopolítico de la educación y destaca la relación entre educación y cultura como factor de conocimiento tanto de sí mismo, como de los demás, de la ciudadanía y del sentimiento de cohesión social y pertenencia a un grupo. Sostiene que la educación no cumpliría su misión si no fuera capaz de formar ciudadanos arraigados a sus respectivas culturas y, no obstante, abiertos a las demás y dedicados al progreso de la sociedad. Los planteamientos resultantes de estos encuentros se han convertido en referentes importantes para profundizar, a nivel de políticas públicas, sobre los objetivos estratégicos de educación para el milenio, así como sobre los problemas y las alternativas de solución para mejorar su calidad y pertinencia.
Así mismo, la idea de una educación para la ciudadanía actualiza la reflexión sobre los fines de la educación en el sentido de la promoción de valores ciudadanos y el refuerzo de las condiciones, tanto para el ejercicio de la ciudadanía como para la participación democrática efectiva en este entorno dinámico, rico en información y en constante cambio. La aproximación hacia una democracia participativa que se propone como nuevo reto educativo se orienta hacia la realización personal de los estudiantes; la creación de condiciones para que estos ejerzan su autonomía y estén en contacto solidario con su medio social, cultural y natural, y logren otorgarle significados personales a su experiencia como personas críticas.

6 Informe Delors, presentado a la Unesco por la Comisión Internacional de Educación para el siglo XXI y conocido bajo el título "La educación encierra un tesoro", plantea una estructura de educación para toda la vida en torno a cuatro aprendizajes fundamentales que se constituyen en pilares del conocimiento: Aprender a conocer, para combinar una cultura general suficientemente amplia con la posibilidad de profundizar los conocimientos en un pequeño número de materias y supone además aprender a aprender para aprovechar las posibilidades que ofrece la educación a lo largo de la vida. Aprender a hacer, para adquirir además de una cualificación profesional, una competencia que capacite al individuo para hacer frente a gran número de situaciones y aprender a trabajar en equipo. Aprender a vivir juntos, para desarrollar la comprensión del otro y la percepción de las formas de interdependencia — realizar proyectos comunes y prepararse para tratar los conflictos- respetando los valores de pluralismo, comprensión mutua y paz. Aprender a ser, para que aflore mejor la propia personalidad y se esté en condiciones de obrar con creciente capacidad de autonomía, de juicio y de responsabilidad personal. 
En este panorama, la reflexión social de la educación como fenómeno cultural y como proceso provoca una planificación intencional de las autoridades educativas de acuerdo con los nuevos ideales y valores, de forma tal que se orienten hacia una sociedad en particular y hacia un tipo de hombre concreto. La educación como fenómeno de carácter dinámico sigue un determinado ritmo que se va ajustando en función de unos fines esperados y depende de múltiples y complejos factores, entre otros, de las relaciones que se establecen entre los actores que intervienen en el proceso educativo y de las funciones que desempeñan cada uno de ellos; de los derechos y obligaciones que se establecen entre uno y otro; de la relación que se produce entre la enseñanza y el aprendizaje y de la función manifiesta de esta relación, en cuanto a la selección, organización y comunicación de los contenidos educativos.

En la educación como proceso es necesario diferenciar la "espontánea" o funcional, de la "sistemática" o planificada. La primera, entendida como aquella que se da naturalmente por el hecho de vivir en sociedad como producto de la participación e interacción comunitaria, y la segunda, como proceso intencional de comunicación de contenidos culturales, en función de un objetivo o fin educativo, y es la que se da en las instituciones educativas. En las sociedades contemporáneas la educación sistemática o planificada se organiza alrededor de dos postulados: el aprendizaje implica una dinámica que va progresiva y gradualmente de lo simple a lo complejo y paralelo con un proceso general de maduración y desarrollo humano.

A nivel internacional fue en la Conferencia de Estocolmo (1972) cuando por vez primera se estableció la recomendación a las naciones de acoger un programa internacional de educación sobre medio ambiente.
Luego, en 1976 y 1977, fueron propuestos el Programa Internacional de Educación Ambiental (PIEA) y elementos de construcción de métodos integradores acordes con la caracterización global del problema ambiental. En 1987 se acogieron estrategias de carácter curricular y se logró consenso sobre el concepto de educación ambiental. En el contexto de la Conferencia de Río, finalmente, se acogieron iniciativas de institucionalización de este contenido en la educación, en un proceso que desde entonces es periódicamente evaluado en sus logros.

La moda instituyente de la educación ambiental llegó al país desde la década de los setenta, como parte del Código Nacional de los Recursos Naturales y Renovables y de Protección del Medio Ambiente por el Inderena. En la Constitución Política del 91 se establecieron parámetros de posibilidad con la inclusión del concepto de “derechos ambientales" y la regulación de competencia por autoridades en la materia. En ese mismo año se definió una "política ambiental para Colombia”. En 1993 se creó el Ministerio del Medio Ambiente, con lineamientos para concertar con el de Educación y apoyar el SINA. Los primeros lineamientos de política educativa ambiental datan de 1995, apoyándose en un Convenio MEN-IDEA de 1992.

La Constitución de 1991 también trajo nuevos retos en relación con el tema de la participación, la construcción de un habitus político en las comunidades y la implementación de sistemas democráticos, carentes en Colombia. Sin embargo, pese a las expectativas de unos y las desilusiones de otros, estos retos se vieron de alguna manera reducidos al entender que el gran problema del país consistía en que era inexistente una cultura ciudadana. Para esto se llevaron grandes campañas gubernamentales y de algunas organizaciones 
sociales sobre la participación en las nuevas figuras político-administrativas, especialmente aquellas relacionadas con la elección popular. Dentro de la ilusión de la participación política de las "minorías" excluidas, especialmente los sectores marginales, se presentó un proceso de instrumentalización de la participación de las comunidades.

Por un lado, las organizaciones sociales y comunitarias locales fueron cooptadas por la institucionalización de sus procesos, que en muchos casos llevan largos procesos sociales, culturales y políticas autogestionadas, y se convirtieron en apéndices en lucha entre ellas por la captación de recursos provenientes de concursos en proyectos gubernamentales y de organizaciones internacionales.

El discurso de la cultura de ciudadanía comenzó a ser implementado en Bogotá desde 1995 durante la alcaldía de Antanas Mockus, con el lema de formar ciudad. Para él la cultura ciudadana se desarrolla bajo la estrategia de:

[...] Impulsar una autotransformación cultural voluntaria y participativa (básicamente consolidar y cambiar hábitos). Pretende también generar una relación armónica de las personas con las leyes y con las reglas culturales de convivencia, buscando obtener una obediencia voluntaria a las normas, una obediencia por convicción (Mockus, 2003).
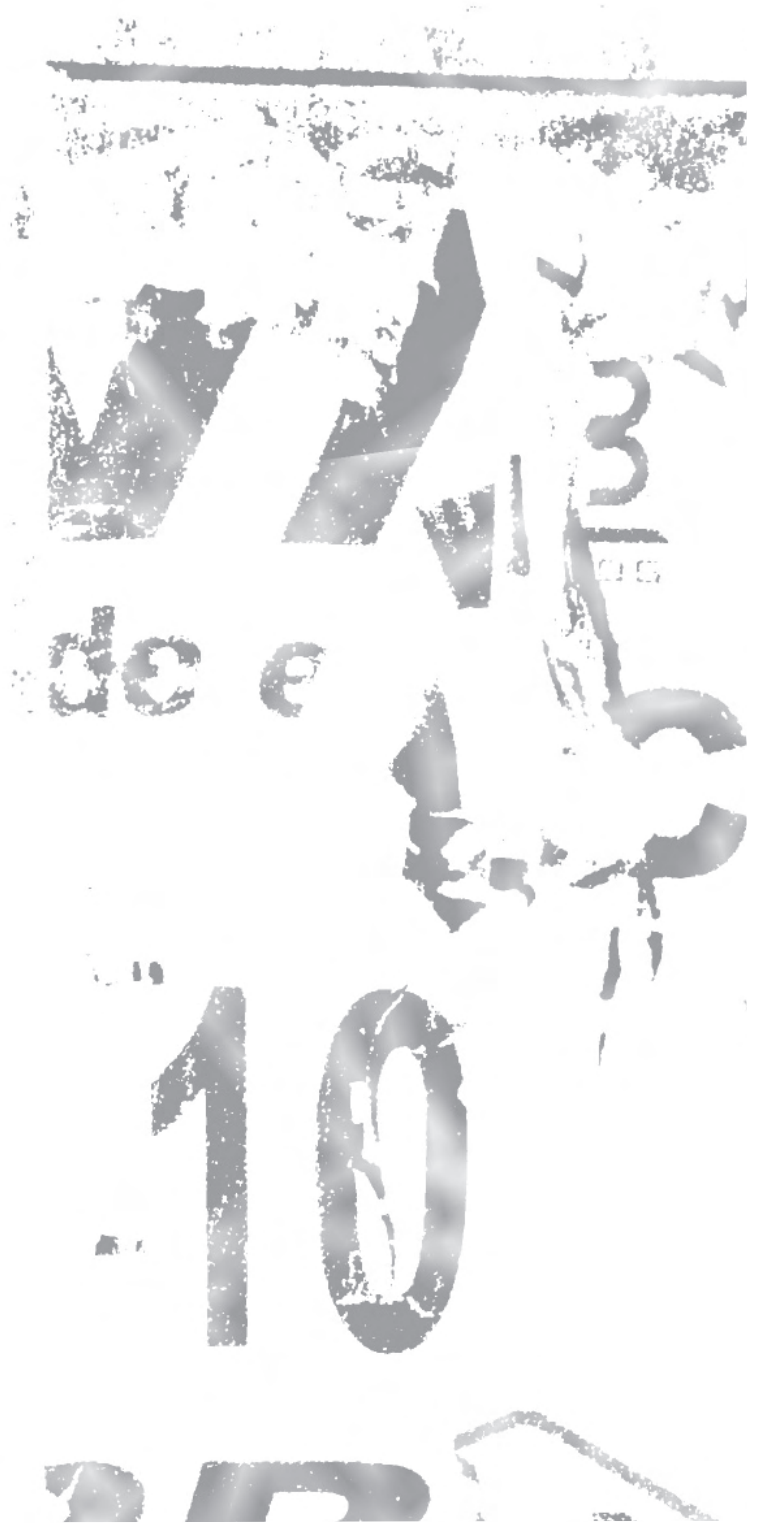
En desarrollo de las estrategias surgieron las campañas de cultura ciudadana, cuyo objetivo primordial consistía en el acatamiento voluntario de la norma para la convivencia en el espacio público. Durante las dos alcaldías de Mockus se enfatizó en que el problema fundamental de la ciudad estaba en que las personas no tenían internalizadas reglas básicas de convivencia, por lo que sus campañas iban dirigidas a lograrlo a través de estrategias como la ley zanahoria, reducir homicidios mediante el desarme; y normas dirigidas exclusivamente al transporte, como respeto a las cebras, que los conductores se pongan el cinturón de seguridad, y la reducción de muertes por accidentes. Pese a las problemáticas estructurales que presentaba la ciudad, como el desempleo, la llegada de miles de familias desplazadas, la inseguridad ligada a escuadrones de la muerte y organizaciones criminales, la incapacidad de fortalecer la seguridad alimentaria, las políticas públicas se empeñaron en incrementar la cultura ciudadana en la perspectiva antes reseñada, omitiendo y desconociendo otras problemáticas, representando así una práctica de gobierno que busca resolver necesidades de orden básico sólo para beneficiar a las clases altas y medias de la ciudad.
Esta política de la cultura ciudadana resultó exitosa y se extendió posteriormente a las administraciones de algunas ciudades del país, de manera adaptativa también fue luego aplicada en Bogotá por los alcaldes sucesores hasta la actualidad, bajo el supuesto de brindar protagonismo a los “ciudadanos” para la participación en las decisiones de la administración distrital. Sin embargo, este tipo de discurso instrumentaliza a las comunidades eufemizando el discurso de la participación mientras continúan excluidos(as) de las decisiones.

Por otra parte, la necesidad de implementar la ciudadanía en el sistema educativo nacional se origina a partir de un estudio de la Asociación Internacional para la Evaluación del Logro Educativo (IEA) denominado Proyecto Educación Cívica, conformada por países entre los que se encontraban Estados Unidos y algunos europeos

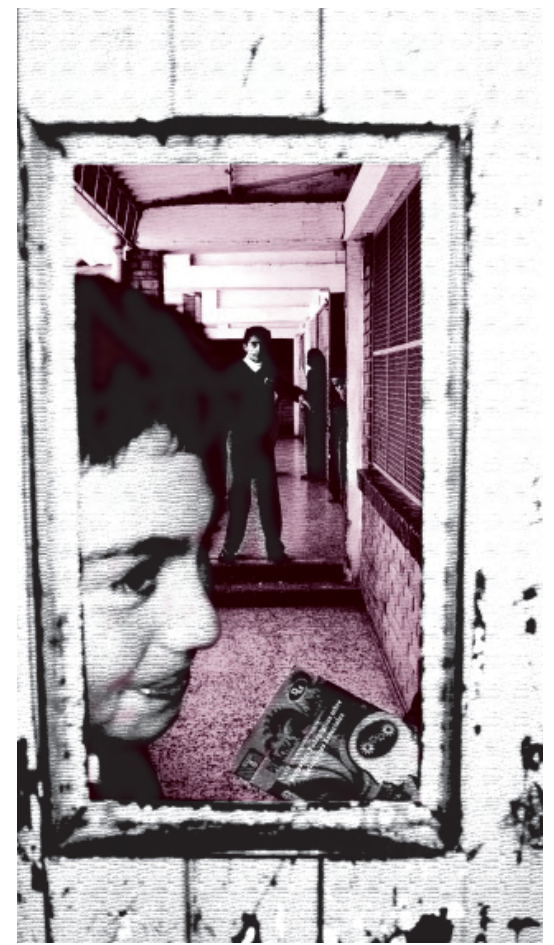


y de América Latina. De ahí se evaluaron los logros en formación cívica, identidad política y formación democrática. Fue entonces cuando se implementó en el sistema educativo colombiano como política pública, además inspirada en las expectativas despertadas por la nueva Carta Magna, desarrollada luego en normas como la Ley 115, el Decreto 1860 y la Resolución 01600 de 1994; también se establecieron los lineamientos curriculares en Constitución Política, democracia, ética y valores humanos en 1998, renovados en 2004 y dados a conocer como Estándares Básicos de competencias ciudadanas, elaborados por el MEN en el primer gobierno de Uribe. En estos la idea de competencia está relacionada con la noción de ser competente a través del saber y el saber hacer, y se agruparon en tres grupos temáticos: convivencia y paz; participación y responsabilidad democrática; pluralidad, identidad y valoración de las diferencias.

Esta educación se centró en la enseñanza de la nueva Carta Constitucional, educación moral centrada en el desarrollo de valores democráticos, la participación de niños y jóvenes en organismos escolares que reproducen los instituidos políticamente, la participación juvenil en asuntos públicos (Pinilla, 2004, 3). La preocupación se centraba en el fomento de valores democráticos y el fortalecimiento de relaciones de convivencia en los lugares públicos y escolares, así como en la participación comunitaria.
Para el caso específico de Bogotá, se aplicó una prueba en los colegios públicos y privados para medir las "competencias" básicas en comprensión y sensibilidad ciudadana de los estudiantes; el énfasis que se aplicó fue el de medir los aspectos axiológicos de la ciudadanía, dejando de lado aspectos ideológicos, político-económicos y socioculturales (Pinilla, 2004, 3), en donde la ciudadanía se puede entender más allá de su aspecto individual. No obstante, va a ser en la administración de Antanas Mockus que se medirá con fuerza la cultura ciudadana, enmarcándose en el paradigma universal hasta entonces predominante que considera la ciudadanía como la manera de actuar moralmente correcta, sustentada en los postulados psicologistas de Kohlberg, donde los valores democráticos se pueden adquirir a través de niveles. Por tanto, para Mockus, la ciudadanía se establece con el trinomio "cultura", ley y moral como componentes reguladores del comportamiento.

Sin embargo, los modelos de intervención para generar cultura ciudadana en un espacio tan particular como el de Bogotá se han revelado insuficientes para lograr en el largo plazo los resultados requeridos sobre un desarrollo deseado. Diversas mediciones apuntan a demostrar estancamientos y retrocesos en el estado previsto de los factores que más frecuentemente se asocian con esta condición: tolerancia, solidaridad, identidad, reconocimiento, etc. No sólo se evidencia la ideologización de las competencias ciudadanas en el enfoque de gobernabilidad urbana, sino que la misma concepción pedagógica de la estrategia de intervención puede resultar afectada por lo que se ha dado en llamar el currículo oculto de los recursos educativos (Ecologistas, 2007). 


\section{Psicopedagogía crítica de los derechos colectivos en temas de educación ambiental}

Se desconocen los espacios que brinda la ciudad como aulas abiertas donde el estudiante puede apropiarse de los conceptos aplicados, como por ejemplo, los humedales, las centrales de Transmilenio, la Central de Abastos, el río como eje articulador y delimitador de la localidad, los diferentes tipos de industrias presentes en la localidad, su comercio y la relación con su entorno, vistos desde el desarrollo y los impactos ambientales que estos generan. ${ }^{7}$

Como marco de referencia para la construcción de una pedagogía ciudadana en materia ambiental, se presenta a grandes rasgos la evolución de los métodos educativos, tradicional, activo y constructivista, desde un enfoque de las pedagogías del conocimiento (Not, 1998).

Las transformaciones pedagógicas suceden cuando las teorías y métodos existentes no permiten resolver los nuevos problemas educativos. Estas renovaciones o "cambios de paradigma" ocurren debido a la mutación de las sociedades y gracias a la capacidad humana para reflexionar críticamente y actuar en consecuencia sobre los diversos aspectos que intervienen en los procesos de enseñanza y aprendizaje. A través de las innovaciones de concepción curricular en la educación básica, los gobiernos nacional y local han buscado en los tres últimos lustros adecuar la organización y planificación de los saberes pedagógicos sobre el medio ambiente a las necesidades de sensibilización de la población escolarizada respecto del significado que estos temas tienen para la sostenibilidad misma de una sociedad tan dependiente del medio natural como es el caso de Colombia. Estas innovaciones han estado recogidas en una serie de propuestas como los estándares curriculares y las competencias básicas, que tienen aspectos en común con el conjunto de los modelos de reorganización de los contenidos de la educación básica.

Al comparar las diversas concepciones se descubre cómo el aprendizaje, desde el enfoque crítico como posibilidad de transformación personal y social, se da en un marco de cooperación y reciprocidad no sólo por las relaciones que se establecen entre los propios estudiantes durante el proceso, sino entre los estudiantes y los educadores, quienes al aprender logran dimensionar los problemas con una mayor comprensión, tanto a nivel académico como de reflexión y acción. En palabras de Freire, nadie educa a nadie, así como tampoco nadie se educa a sí mismo, los hombres se educan en comunión, y el mundo es el mediador (Freire, 2005, 92).

7 Sánchez, J. C., Formación de cadenas tróficas artificiales para el manejo y disposición de los residuos sólidos orgánicos en la IED INEM Francisco de Paula Santander, proyecto premiado en la convocatoria Innovación Educativa y Experiencia Pedagógica, Bogotá, IDEP, 2008. 
La propuesta de construcción de una pedagogía ciudadana en materia ambiental se basa en un enfoque de educación que prepare a los agentes sociales y a la sociedad en conjunto para interiorizar pautas de convivencia más justas y en armonía con el medio natural. Este producto requiere un sujeto social con un habitus de participación política y con competencias no mercantiles que le permitan revalorizar las relaciones individuo-naturaleza-sociedad.

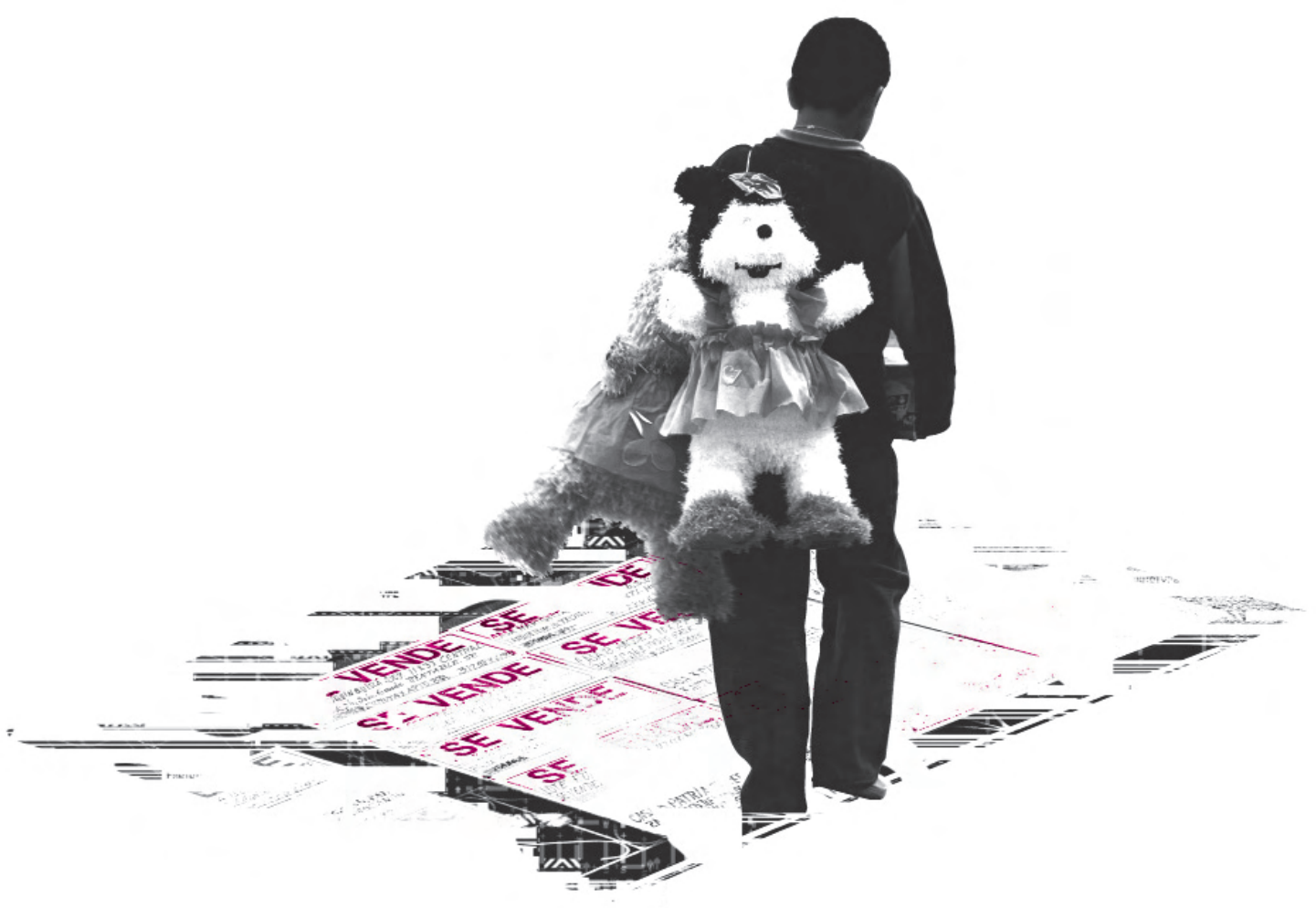


Un primer objetivo de una estrategia educativa de tipo ambiental está ligado a la afirmación de una disposición de tipo ciudadano y de tipo ambiental. A eso se le suele llamar ciudadanía ambiental o ecociudadanía: este concepto nace desde las Naciones Unidas, y en el caso de América Latina se desarrolla bajo el proyecto Ciudadanía Ambiental, del PNUMA, en el cual participan la UICN y su Comisión de Educación y Comunicación (CEC). Parten de caracterizar como principales preocupaciones temas como la escasez del agua, la delimitación de las aguas internacionales, la biodiversidad, el cambio climático y la capa de ozono. Entre las reivindicaciones que se tienen están:

- Los derechos a la vida, al desarrollo sustentable y al ambiente.

- Los deberes ambientales, diferenciados según roles sociales.

- La participación real para defender los derechos y llevar a la práctica los deberes ambientales.

Una de las definiciones de la ciudadanía ambiental consiste en entenderla como:

La integración dinámica entre el reconocimiento de los derechos al ambiente y a la vida, los deberes diferenciados de ciudadanos y ciudadanas frente al ambiente y el desarrollo sustentable, y la participación activa de todos y todas para defender sus derechos y ejercitar cotidianamente sus respectivas responsabilidades, en un marco ético y de valoración de la vida en todas sus manifestaciones (Tréllez, E, 2006).
Las anteriores precisiones y propuestas, la economía de estado fijo y el desarrollo cualitativo, implican un drástico y profundo cambio del modelo de desarrollo actual y se convierten, tal vez, en el objetivo fundamental cuando de educación ambiental se trata. De hecho, así lo expone el cuarto principio de la Educación Ambiental inscrito en la Política Nacional de Educación Ambiental: ofrecer las herramientas para una reflexión crítica sobre los presupuestos epistemológicos y éticos que soportan el paradigma dominante de desarrollo, con el fin de que a partir de esa reflexión se pueda construir un modelo social y ambientalmente sustentable.

En el Foro Educativo Distrital 2008, uno de los elementos innovadores lo constituye la propuesta de evaluación integral, dialógica y formativa que se plantea en la reorganización de la educación básica y media, conforme a una estructuración de cinco ciclos y períodos académicos consecutivos. Se trata de una propuesta diferenciable de la establecida en la Ley General de la Educación y de la Evaluación de Competencias propuesta por el Ministerio de Educación Nacional. Al respecto de la gradualidad cíclica es preciso entenderla como un modelo analíticamente útil, lo cual no debe confundirse con el reconocimiento de que el desarrollo humano es un continuum sobre el cual no es generalizable decir cuándo comienzan y terminan etapas sucesivas por las que todos los individuos pasan, ya que en ese movimiento particular influyen diversos factores singulares. De ahí que se acepte que cada ser humano tiene su propio ritmo y se asuma esta consecuencia sobre los modelos pedagógicos. 


\section{Perspectivas de innovación y transformación de la educación ambiental escolar en Bogotá}

Los factores que se sugieren a continuación para el desarrollo de una propuesta escolar orientada al fortalecimiento de la educación ambiental en el espacio social del Distrito, buscan estar en concordancia con la reorganización por ciclos y su marco evaluativo integral, dialógico y formativo. Aun no siendo excluyentes se estiman como de favorable incidencia en el tipo de educación ambiental de los derechos colectivos.

\section{Matriz de competencias mínimas para fortalecer la dimensión ambiental ${ }^{8}$}

\section{Contenidos de competencias para Primer Ciclo}

\begin{tabular}{c|c|c}
\hline Cognitivas & $\begin{array}{c}\text { Emocionales y } \\
\text { comunicativas }\end{array}$ & $\begin{array}{c}\text { Participación ciudadana desde } \\
\text { derechos (mínimos de acción) }\end{array}$ \\
\hline $\begin{array}{c}\text { Comprendo que todos los seres vivos } \\
\text { necesitamos energía para vivir; que esta } \\
\text { proviene de los alimentos, la luz y el calor del } \\
\text { Sol, y que la convierto en movimiento, calor, } \\
\text { fuerza y vitalidad }\end{array}$ & $\begin{array}{c}\text { Me intereso por los temas } \\
\text { ambientales }\end{array}$ & $\begin{array}{c}\text { Participo en actividades que reflejen manejo } \\
\text { responsable de los recursos del plantel } \\
\text { educativo }\end{array}$ \\
\hline $\begin{array}{c}\text { Comprendo que mi vida transcurre en } \\
\text { determinados espacios: la casa, el colegio, } \\
\text { el barrio, y que comparto esos espacios con } \\
\text { otras personas y otros seres vivos }\end{array}$ & $\begin{array}{c}\text { Empiezo a ser consciente } \\
\text { de problemas ambientales }\end{array}$ & $\begin{array}{c}\text { Identifico y respeto a los semejantes y las } \\
\text { diferencias entre los demás, yo y otros seres } \\
\text { vivos }\end{array}$ \\
\hline $\begin{array}{c}\text { Comprendo que no todos los seres vivos son } \\
\text { iguales; comparten algunas características que } \\
\text { los hacen pertenecer a una misma clase, por } \\
\text { ejemplo las aves, los peces; y comprendo que } \\
\text { no todos pueden vivir en los mismos sitios y } \\
\text { no todos comen lo mismo }\end{array}$ & $\begin{array}{c}\text { Soy capaz de utilizar } \\
\text { habilidades orales, escritas } \\
\text { artísticas para comunicar } \\
\text { información sobre el } \\
\text { ambiente }\end{array}$ & $\begin{array}{c}\text { Comprendo la importancia de normas básicas } \\
\text { para el cuidado de mí mismo, de los seres } \\
\text { vivos y de mi entorno }\end{array}$ \\
\hline
\end{tabular}

8 Por razones de espacio se omite la presentación exhaustiva de la matriz. 


\begin{tabular}{c|c|c}
\hline Cognitivas & $\begin{array}{c}\text { Emocionales y } \\
\text { comunicativas }\end{array}$ & $\begin{array}{c}\text { Participación ciudadana desde } \\
\text { derechos (mínimos de acción) }\end{array}$ \\
\hline Eje cognitivo & Eje comunicativo & Eje acción \\
\hline $\begin{array}{c}\text { Comprendo los diferentes usos que se le dan } \\
\text { al agua en el contexto cotidiano, la utilidad } \\
\text { del suelo y el agua para las plantas y para } \\
\text { algunos animales. Comprendo la relación } \\
\text { del aire como medio por el que se desplazan } \\
\text { ciertos animales y medios de transporte, y su } \\
\text { importancia en la vida }\end{array}$ & & \\
\hline $\begin{array}{c}\text { Comprendo que vivo con mi familia, que } \\
\text { tengo amigos y parientes que viven en la } \\
\text { misma ciudad o en otro lugar, y que todos } \\
\text { nosotros usamos cosas parecidas en la vida } \\
\text { cotidiana }\end{array}$ & & \\
\hline
\end{tabular}

\section{Contenidos de competencias para Cuarto Ciclo}

\begin{tabular}{|c|c|c|}
\hline Cognitivas & $\begin{array}{l}\text { Emocionales y } \\
\text { comunicativas }\end{array}$ & $\begin{array}{l}\text { Participación ciudadana desde } \\
\text { derechos (mínimos de acción) }\end{array}$ \\
\hline Eje cognitivo & Eje comunicativo & Eje de acción \\
\hline $\begin{array}{l}\text { Comprendo que todos los productos que } \\
\text { existen en el mercado han sido producidos } \\
\text { usando fuentes de energía renovables y no } \\
\text { renovables, y que los hábitos de consumo } \\
\text { actuales, estimulados por los medios de } \\
\text { comunicación, generan cada vez mayor } \\
\text { agotamiento de las fuentes energéticas. } \\
\text { Comprendo que el uso de fuentes energéticas } \\
\text { alternativas beneficia la sostenibilidad en el } \\
\text { planeta }\end{array}$ & $\begin{array}{l}\text { Reconocer y asumir la } \\
\text { responsabilidad de mis } \\
\text { acciones y de la toma de } \\
\text { decisiones }\end{array}$ & $\begin{array}{c}\text { Participo en discusiones que involucran } \\
\text { predecir posibles efectos relacionados con } \\
\text { el uso de artefactos, procesos y sistemas } \\
\text { tecnológicos }\end{array}$ \\
\hline $\begin{array}{l}\text { Comprendo que todo lo que me rodea, } \\
\text { incluso mi vivienda, proviene de recursos } \\
\text { naturales extraídos. Comprendo que } \\
\text { la concentración de las personas en } \\
\text { determinadas regiones del país conlleva la } \\
\text { transformación, agotamiento y deterioro del } \\
\text { medio ambiente local. Comprendo además } \\
\text { que para suplir las necesidades locales se } \\
\text { están usando recursos de otras regiones, } \\
\text { aumentando el deterioro ambiental }\end{array}$ & $\begin{array}{l}\text { Realizar juicios basados en } \\
\text { la información disponible } \\
\text { sobre problemáticas } \\
\text { ambientales y efectos en el } \\
\text { planeta y en los seres vivos }\end{array}$ & $\begin{array}{l}\text { Asumo posturas frente a conflictos de } \\
\text { intereses y de derechos en conflicto (ejes } \\
\text { económicos, sociales, ambientales, } \\
\text { éticos, etc,) }\end{array}$ \\
\hline
\end{tabular}




\begin{tabular}{|c|c|c|}
\hline Cognitivas & $\begin{array}{l}\text { Emocionales y } \\
\text { comunicativas }\end{array}$ & $\begin{array}{l}\text { Participación ciudadana desde } \\
\text { derechos (mínimos de acción) }\end{array}$ \\
\hline Eje cognitivo & Eje comunicativo & Eje de acción \\
\hline $\begin{array}{l}\text { Comprendo que todos los recursos provienen } \\
\text { de los diversos ecosistemas y, en esa medida, } \\
\text { como materias primas de los productos que } \\
\text { consumimos, inciden en menor o mayor } \\
\text { grado sobre la conservación del ecosistema. } \\
\text { Comprendo que los ecosistemas, como } \\
\text { sistemas abiertos, dependen de intercambios } \\
\text { internos y externos de energía, de nutrientes } \\
\text { entre las especies asociadas a estos: plantas, } \\
\text { animales y micrororganismos }\end{array}$ & $\begin{array}{l}\text { Entender cómo la } \\
\text { problemática de mi } \\
\text { localidad hace parte del } \\
\text { problema ambiental } \\
\text { regional, nacional y global }\end{array}$ & $\begin{array}{c}\text { Identifico en otras comunidades humanas su } \\
\text { interacción con el ambiente y el impacto de } \\
\text { sus actividades }\end{array}$ \\
\hline \multirow{2}{*}{$\begin{array}{l}\text { Comprendo que no todos los recursos están } \\
\text { disponibles de igual forma para todos, que } \\
\text { sólo unos tienen capacidad económica de } \\
\text { acceder a dichos recursos, que hay recursos } \\
\text { que pertenecen a todos, que algunos de } \\
\text { ellos están contaminados y agotados y que } \\
\text { su recuperación depende de acciones para } \\
\text { subsanar o evitar que el deterioro continúe }\end{array}$} & $\begin{array}{l}\text { Entender las relaciones } \\
\text { con el territorio y los } \\
\text { recursos de nuestras } \\
\text { culturas ancestrales }\end{array}$ & \\
\hline & $\begin{array}{l}\text { Apreciar acciones de } \\
\text { individuos y grupos } \\
\text { (locales, nacionales y } \\
\text { mundiales) a favor del } \\
\text { medio ambiente }\end{array}$ & \\
\hline \multirow{3}{*}{$\begin{array}{l}\text { Comprendo que las diversas acciones que se } \\
\text { llevan a cabo en la ciudad, las domésticas e } \\
\text { industriales, deterioran la calidad ambiental } \\
\text { de la ciudad y de otros lugares aledaños. } \\
\text { Comprendo también que la mayoría de los } \\
\text { bienes y servicios que utilizamos en la ciudad } \\
\text { provienen de otras partes. }\end{array}$} & $\begin{array}{c}\text { Describir el impacto } \\
\text { ambiental de los países } \\
\text { desarrollados y de los en } \\
\text { vía de desarrollo }\end{array}$ & \\
\hline & $\begin{array}{c}\text { Reconocer las } \\
\text { características únicas de } \\
\text { Bogotá y de Colombia, y } \\
\text { la oferta local, regional y } \\
\text { nacional de recursos }\end{array}$ & \\
\hline & $\begin{array}{c}\text { Explorar y analizar } \\
\text { acciones para solucionar } \\
\text { los problemas ambientales } \\
\text { a nivel local y nacional }\end{array}$ & \\
\hline
\end{tabular}


Matriz de conocimientos mínimos generales. Eje cognitivo

\begin{tabular}{|c|c|}
\hline $\begin{array}{c}\text { Componente de la dimensión } \\
\text { ambiental }\end{array}$ & $\begin{array}{l}\text { Conocimientos mínimos por componente: } \\
\text { contexto general }\end{array}$ \\
\hline Fuentes energéticas y actividad humana & $\begin{array}{l}\text { - Fuentes de energía renovables y no renovables } \\
\text { - Sistemas de transporte, consumo de energía, emisión de CO2 } \\
\text { - Industria y energía. Demanda de energía } \\
\text { - Alternativas energéticas, modelos de desarrollo y estilos de vida, medios } \\
\text { de comunicación y consumo } \\
\text { - Flujos de energía en sistemas naturales y procesos productivos }\end{array}$ \\
\hline Población y medio ambiente & $\begin{array}{l}\text { - Seguridad alimentaria } \\
\text { - Demografía } \\
\text { - Acceso equitativo a bienes y servicios ambientales } \\
\text { - Equilibrio sociedad-naturaleza } \\
\text { - Escasez de recursos } \\
\text { - Impacto humano sobre ambiente, economía y medio ambiente } \\
\text { - Política y medio ambiente } \\
\text { - Territorio y medio ambiente }\end{array}$ \\
\hline Biodiversidad y distribución geográfica & $\begin{array}{l}\text { - Ecosistemas: flujos de energía, flujos de nutrientes, cadenas alimentarias, } \\
\text { nichos, resiliencia ecosistémica, límites ecosistémicos } \\
\text { - Especies: diversidad, riqueza, evolución, extinción de especies } \\
\text { - Poblaciones: distribuciones geográficas, disminución de poblaciones, } \\
\text { extinción de poblaciones }\end{array}$ \\
\hline Oferta ambiental y equidad & $\begin{array}{l}\text { - Agua: disponibilidad, contaminación, usos, acceso } \\
\text { - Suelo: erosión, contaminación, pérdida } \\
\text { - Atmósfera: contaminación, calentamiento global, efecto invernadero } \\
\text { - Biodiversidad: agotamiento, acceso equitativo a la oferta ambiental }\end{array}$ \\
\hline Reto urbano y huella ecológica & $\begin{array}{l}\text { - Migración de poblaciones, concentración de población } \\
\text { - Disponibilidad y demanda de recursos naturales en las ciudades } \\
\text { - } \text { Biodiversidad en las ciudades } \\
\text { - Huella ecológica de las ciudades: contaminación atmosférica, contaminación } \\
\text { - hídrica, residuos sólidos y líquidos } \\
\text { - Huella ecológica individual } \\
\text { - Transeza y marginalidad } \\
\text { - Desastres naturales }\end{array}$ \\
\hline
\end{tabular}




\begin{tabular}{c|c|c}
$\begin{array}{c}\text { Componente de la dimensión } \\
\text { ambiental }\end{array}$ & \multicolumn{1}{c}{$\begin{array}{c}\text { Conocimientos mínimos por componente: } \\
\text { contexto general }\end{array}$} \\
\hline & $\begin{array}{l}\text { - Procesos lineales } \\
\text { - Procesos sistémicos }\end{array}$ \\
Procesos productivos - ambiente & - Componentes de los sistemas productivos \\
& - Consumo, oferta, demanda, obsolescencia planificada y percibida \\
& - Fuentes y sumideros, contaminación, materias primas, residuos \\
& - Sociedad e industria \\
& - Ambiente y agricultura \\
\hline
\end{tabular}

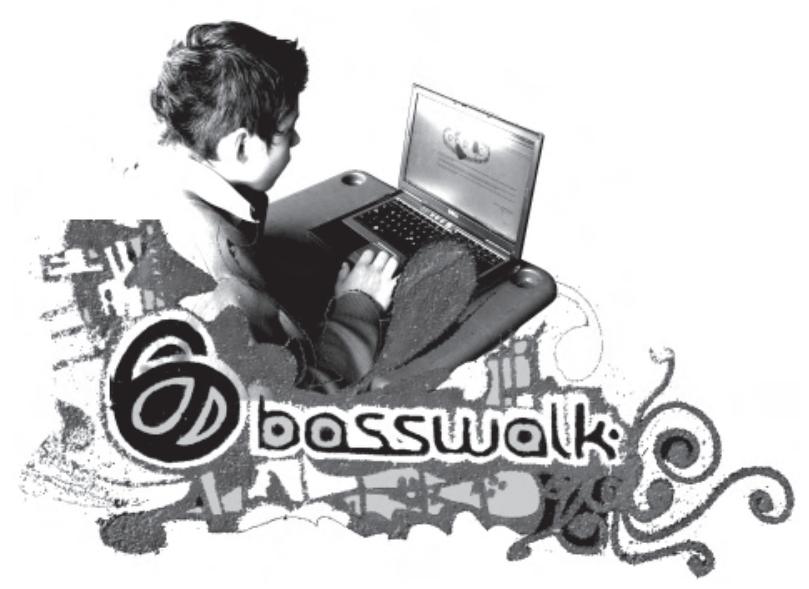


La educación ambiental en el marco de la responsabilidad y de la solidaridad requiere abordar la crisis planetaria desde dos importantes componentes: por un lado, el gran crecimiento poblacional que engrosa la masa del subdesarrollo y de la pobreza y, por el otro, el de los graves procesos de degradación ambiental que afectan a los ecosistemas en los ámbitos locales, regionales y globales, y que amenazan con alterar la capacidad de autorregulación del planeta.

Otros factores que requieren ser tomados en cuenta en la educación hacen referencia a la distancia cada vez mayor entre la sociedad y la naturaleza, a una sobrevaloración de la sociedad de consumo irracional que sobrepone el tener al ser; a una pérdida de calidad de vida de la población en general; a la degradación de los sistemas ambientales representada por procesos globales y regionales como la destrucción de la capa de ozono, la pérdida de biodiversidad, la disminución de las tierras fértiles, el cambio climático, la amenaza e incertidumbre de los desechos peligrosos, la disminución del acceso al agua potable, la contaminación de las fuentes y recursos hídricos y la deforestación, todos estos considerados factores que explican la centralidad de la definición "sociedad del riesgo", en oposición a la era del bienestar.

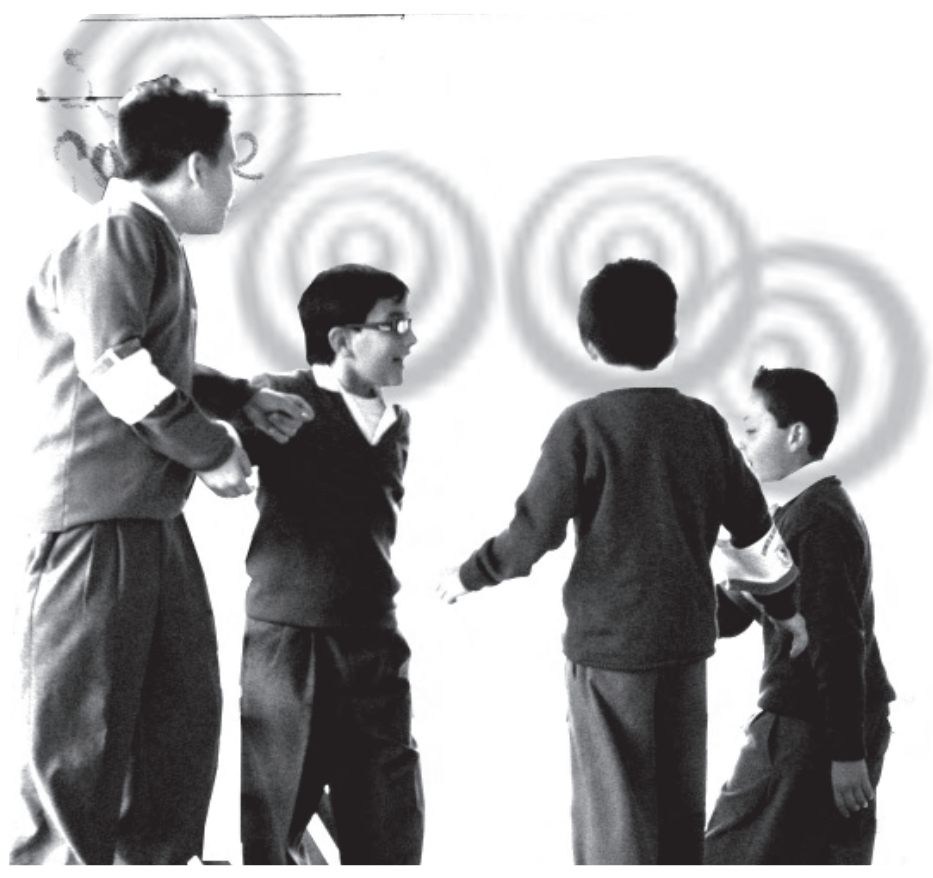




\section{A manera de conclusión: posibilidades de la ciudadanía ambiental}

Por lo anterior, a partir de la visión global de los derechos y deberes relacionados con el ambiente y el desarrollo sustentable se va conformando gradualmente el concepto de ciudadanía ambiental a nivel planetario. Este proyecto podría hacer parte de la estrategia de pedagogía ciudadana para la transformación cultural en la ciudad de Bogotá en los próximos años. Para aportar al tema de la comprensión del concepto se pueden manejar tres elementos de base cuya articulación conforma la ciudadanía ambiental:

- Los derechos a la vida, al desarrollo sustentable y al ambiente.

- Los deberes ambientales, diferenciados según roles sociales.

- La participación real para defender los derechos colectivos y llevar a la práctica los deberes ambientales.

La formación de ciudadanía ambiental puede darse a través de la fusión activa de estos factores interactuantes, cuyas mutuas relaciones deben contar con un indispensable sustento ético y de construcción de valores. Es posible decir, entonces, que se forma ciudadanía ambiental, entre otras acciones, cuando:

- Se apoya el reconocimiento y defensa de los derechos a la vida, al desarrollo sustentable y a un ambiente sano.

- Se promueve el ejercicio de los deberes ambientales, diferenciando a los sectores y grupos, en sus respectivas responsabilidades.
- Se crean, refuerzan o promueven mecanismos e instrumentos efectivos de participación ciudadana para el ejercicio de los derechos y deberes ambientales, en un marco ético y de responsabilidad.

- Se forma a los ciudadanos y ciudadanas para participar directamente en procesos de gestión ambiental, orientados a la defensa de la vida y del patrimonio social y cultural.

- Se realizan actividades de participación efectiva de la ciudadanía en dichos procesos, a diversos niveles y con diferentes responsabilidades.

En este sentido, una definición de ciudadanía ambiental podría ser la siguiente:

La ciudadanía ambiental es la integración dinámica entre el reconocimiento de los derechos al ambiente y a la vida, los deberes diferenciados de ciudadanos y ciudadanas frente al ambiente y el desarrollo sustentable, y la participación activa de todos y todas para defender sus derechos y ejercitar cotidianamente sus respectivas responsabilidades, en un marco ético y de valoración de la vida en todas sus manifestaciones (Tréllez, 2005).

En otras palabras, podemos decir que necesitamos educarnos para ser:

- Ciudadanos y ciudadanas ambientales con un sustento ético y un conjunto de valores que permitan la construcción de nuevos seres humanos, propiciando la relación armónica entre la sociedad y la naturaleza, en la perspectiva del desarrollo sustentable. 


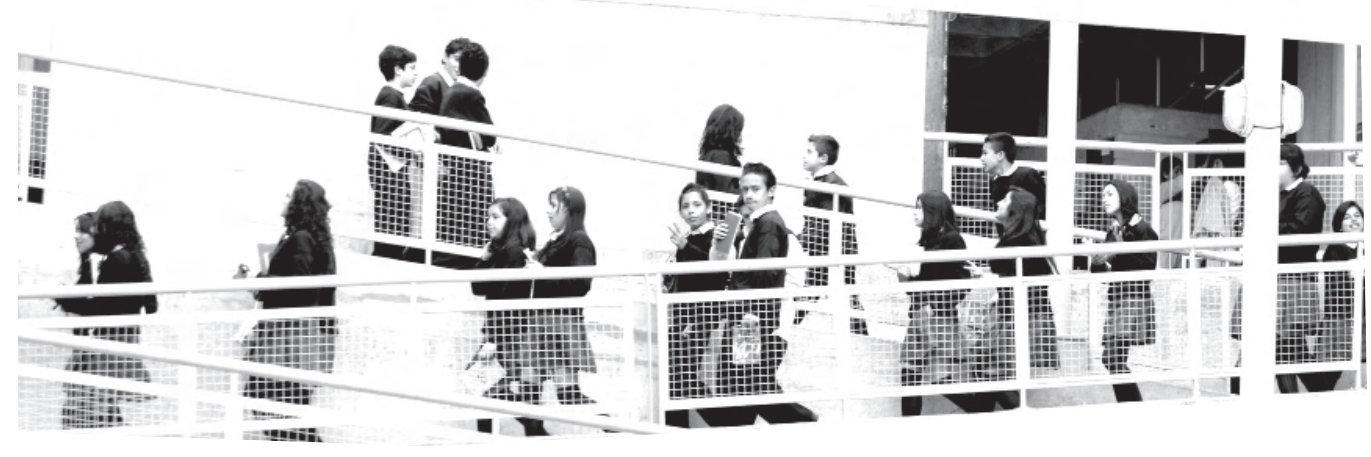

- Ciudadanos y ciudadanas ambientales, críticos y conscientes, que comprendan, se interesen, reclamen, y exijan sus derechos ambientales, estando a la vez dispuestos a ejercer sus propias responsabilidades ambientales.

- Ciudadanos y ciudadanas ambientales capaces de organizarse y participar en la dirección de su propia vida, adquiriendo también poder político comunitario, local, regional, nacional, junto con una gran capacidad de cambio colectivo.
- Ciudadanos y ciudadanas ambientales comprometidos con un cambio profundo de mentalidad, de conceptos y de valores respecto al ambiente.

- Ciudadanos y ciudadanas ambientales con capacidad de vigilancia y participación en la aplicación de las políticas públicas, con proyectos propios para lograr un manejo de los recursos naturales, con una racionalidad social equitativa y un entorno más habitable. 
Pinilla Díaz, A. "La búsqueda de la ciudadanía: estrategias oficiales para desarrollar "habilidades" ciudadanas", en Plegable n 9 9, Sociales, Bogotá, Cedecs, 2004.

Secretaría de Educación Distrital. Foro educativo distrital 2008: documentos para el debate: evaluación integral para la calidad de la educación, Bogotá. 2008.

Trelléz Solís, E. El concepto de ciudadanía ambiental y su formación en América Latina. (Consulta en 10-11-2008). (Disponible en http://www.piramide.org.pe/Piramide/piramide_articulos).

UNESCO IBE. Conferencia Internacional de Educación y propuestas de acción, CIE, 2001 (Disponible en http://www. ibe.unesco.org).

VeGA, C. RenÁn. El pensamiento crítico en un mundo incierto, Caracas, 2008 (Disponible en http://www.rebelion.org/ noticia.php?id=71788).

Viceministerio del Medio Ambiente. Sistema Nacional Ambiental, SINA [recuperado de Viceministerio de Ambiente, consulta en 10-11-2008] (Disponible en http://web.minambiente.gov.co/html/vicesina/sistemanal/home.htm).

Zimmermann, M. Ecopedagogía para el nuevo milenio, Bogotá, Ecoe, 2001.

Willches-Chaux, G. La letra con risa entra: ¿Y... qué es eso de Educación Ambiental?, Bogotá, 1996. 